\title{
Estado nutricional, parasitismo intestinal \\ y sus factores de riesgo en una población vulnerable del municipio de Iza (Boyacá), Colombia año 2013
}

\author{
Nutritional status, intestinal parasitism \\ and their risk factors in a vulnerable \\ population in the municipality \\ of Iza (Boyaca), Colombia 2013
}

\begin{abstract}
The aim of this study was to establish the nutritional status and intestinal parasitism and their risk factors in pregnant women, elderly people and children under 5 years old. Each one of the participants was given a survey, their nutritional status values were taken(weight and size), as were measurements of indicators such as haemoglobin, seric iron, ferritin, tranferrin, reactive protein $C$, and a test for intestinal parasites, followed by a medical management plan. The risk factors for intestinal parasitism found, were bad hygiene habits and the lack of access to basic public services. In the elderly, it was found that $22 \%$ were overweight and $16.5 \%$ suffered from obesity. In the group of boys and girls, $15.2 \%$ were overweight or obese and $15.2 \%$ presented some risk of acute malnutrition. Moreover, the elderly presented $26.4 \%$ prevalence of mild or moderate anaemia, and the children $14.9 \%$, in both cases co-related to the concentrations of iron and ferritin. There was $65.3 \%$ prevalence of intestinal parasites. Some of the parasites found were: Entamoeba coli, Blastocystis hominis and Entamoeba histolytica/E. dispar. It is important to reinforce the programmes of food security to lower the malnutrition rates. In spite of having found a high percentage of intestinal parasites, no statistically significant relationship with malnutrition and its factors was found.
\end{abstract}

Key words: vulnerable population, intestinal parasitism, malnutrition, iron deficiency, anaemia.
Ana Yervid Rodríguez (1) Janneth Marcela Camacho (2) Cesar Mauricio Baracaldo (2) Universidad Pedagógica y Tecnológica de Colombia (UPTC) - Tunja-Boyacá. Colombia Escuela de Medicina, Facultad de Ciencias de la Salud. (2) Grupo de Investigación Atención Primaria en Salud. UPTC. Escuela de Medicina, Facultad de Ciencias de la Salud. Colombia.

Dirigir la correspondencia a: Profesora Ana Yerid Rodríguez Grupo de Investigación Atención Primaria en Salud. UPTC. anayervid.rodriguez@uptc.edu.co

Este trabajo fue recibido el 13 de Diciembre de 2014 aceptado con modificaciones el 26 de Marzo de 2015 y aceptado para ser publicado el 5 de Octubre de 2015.

\section{INTRODUCCIÓN}

Entre los problemas prioritarios de salud pública en los países en vías de desarrollo están la desnutrición y el parasitismo intestinal, situación nutricional de una población que refleja en gran medida su nivel de bienestar y puede ser utilizada para identificar inequidades que podrían afectar su productividad. Asimismo la desnutrición crónica, identificada por el retraso en el crecimiento lineal o la talla baja de los niños, se asocia con menor desempeño escolar, menor productividad y menor ingreso laboral en la vida adulta (1). La deficiencia de micronutrientes, particularmente de zinc, eleva el riesgo de sufrir de talla baja, mientras que la de otros nutrientes como el hierro y el yodo afectan el desarrollo cognitivo y

motor de los niños (2). Cuando estas deficiencias ocurren en el período gestacional o en los primeros 2 años de vida, etapa crítica para el crecimiento y el desarrollo, los daños ocurridos son irreversibles (3). Estudios de intervención realizados en América Latina y otras partes del mundo han mostrado que la inversión en nutrición durante esta etapa conlleva beneficios que van desde un mejor desempeño escolar hasta ingresos más altos en la vida adulta, con implicaciones significativas para la productividad del país (4). Por este motivo, aunque el bienestar nutricional es importante a lo largo de la vida, la nutrición de los niños menores de 5 años, en gran medida refleja el potencial de la futura generación en una sociedad (5).

Según la Encuesta Nacional de la Situación Nutricional 
en Colombia (ENSIN-2010). El país vive una transición demográfica y nutricional debido a que su población presenta al mismo tiempo problemas por déficit y exceso de peso en los diferentes grupos de edad. En niños aún se observa un retraso del crecimiento (desnutrición crónica), en especial en aquellos en nivel 1 y 2 del Sistema de Identificación de Potenciales Beneficiarios de Programas Sociales (SISBEN). En adultos la obesidad es mayor en mujeres y a mayor nivel del SISBEN, en la zona urbana, sin diferencias por etnia ni escolaridad. La prevalencia de obesidad abdominal es mayor en hombres en niveles de SISBEN más altos en la zona urbana y en las mujeres con menor nivel educativo (6).

El exceso de peso en la población gestante muestra una tendencia al incremento, a pesar de los esfuerzos realizados para su prevención (Política de Seguridad Alimentaria, Ley de Obesidad, Guías alimentarias para la población Colombiana) (7).

Las parasitosis intestinales son actualmente uno de los principales problemas médico sociales en el mundo, principalmente en los países en vías de desarrollo, donde las servicios básicos sanitarios, la educación y cultura higiénica son inadecuadas, favoreciendo su permanencia en estas regiones (8). Estas enfermedades se presentan sin distinción de edad, raza, género, estado económico o situación geográfica, incluso su frecuencia puede variar de una región a otra, haciéndolas difíciles de controlar, no sólo por su gran difusión sino por los diversos factores que intervienen en su propagación tales como ambientales, culturales inmunológicos y genéticos $(9,10)$. A pesar que la tasa de mortalidad de las enfermedades parasitarias es baja, la morbilidad es alta, deteriorando la calidad de vida de quienes lo padecen, causando trastornos como pérdida de peso, retardo del crecimiento, pérdida de apetito, problemas cognitivos y mala absorción de nutrientes. En Colombia al igual que otros países en vía de desarrollo, se han realizado numerosos esfuerzos para controlar el desarrollo de las parasitosis (campañas educativas, tratamiento antiparasitario, infraestructura sanitaria), sin embargo su prevalencia permanece alta especialmente en las zonas económicamente deprimidas. Lo que sugiere reforzar las políticas hacia la educación en la comunidad centrándose en aspectos como el conocimiento de los mecanismos de transmisión de estos parásitos, de los hábitos higiénicos personales (como lavarse las manos antes de la preparación de los alimentos, antes de comer y después de defecar, no caminar descalzos, hervir el agua entre otras) y de la vivienda (11,12).

El objetivo de la presente investigación fue establecer el estado nutricional, los factores de riesgo y la prevalencia de parásitos intestinales en una población vulnerable del municipio de Iza.

\section{SUJETOS Y METODOS}

Población estudiada: el municipio de Iza tiene una población de 2013 habitantes de los cuales 52\% corresponde a población rural y 48\% a urbana, de los cuales 1532 pertenecen al régimen subsidiado de acuerdo al puntaje de clasificación del SISBEN; de estos la población de adultos mayores fue 244, niños menores de cinco años 163 y gestantes 7. Se realizó un estudio de tipo descriptivo de corte transversal, en donde la muestra estuvo conformada por 91 adultos mayores, 46 niños menores de cinco años y 7 mujeres gestantes. Inicialmente para seleccionar la muestra de niños menores de cinco años, se realizó la identificación de la población en riesgo de desnutrición, teniendo en cuenta el tamizaje de su condición nutricional, en los registros de control de crecimiento y desarrollo en la entidad de salud del municipio, la Empresa Social del Estado
(E.S.E) Luis Patiño del Municipio de Iza- Boyacá- Colombia. Para el caso de los adultos mayores fueron convocados la totalidad y se incluyeron en el estudio aquellos que voluntariamente quisieron participar, manifestación hecha mediante el consentimiento informado. De esta forma la muestra estuvo conformada por 144 personas.

Ética: en la investigación se siguieron las normas éticas para trabajos en la comunidad, tratadas en la resolución 008430 de 1993 del Ministerio de Salud de Colombia (13), por otra parte fue aprobada previamente por el Comité de Etica de la Universidad Pedagógica y Tecnológica de Colombia - Tunja.

Encuesta: la encuesta fue diseñada teniendo en cuenta los objetivos del proyecto y fue estandarizada y validada en el grupo de investigación de Atención Primaria en Salud de la Universidad Pedagógica y Tecnológica de Colombia - Tunja, con los semilleros de investigación pertenecientes al grupo.

Para la aplicación, se impartió una sesión informativa sobre los alcances del proyecto y educativa sobre parasitismo intestinal a los adultos mayores, padres o tutores, a las madres comunitarias responsables de cada uno de los hogares, y a las mujeres gestantes. Luego se solicitó por medio de consentimiento informado quienes voluntariamente deseaban participar en el estudio y se les aplicó una encuesta que hacía referencia a los siguientes aspectos: caracterización socioeconómica, hábitos alimentarios, hábitos higiénicos y condiciones de la vivienda entre otros. En el caso de los niños menores de cinco años se preguntó también aspectos relacionados con la lactancia materna, crecimiento y desarrollo; información que fue obtenida de sus madres.

Estudio nutricional: para la evaluación del estado nutricional se tomó el peso utilizando una balanza digital grado médico (RICE LAKE RL-440HH, precisión de 1g), Balanza electrónica pesa bebe (marca Detecto, precisión $1 \mathrm{mg}$ ). La estatura se midió con un tallímetro en madera con pieza movible y mediciones en centímetros con exactitud de un milímetro. El estado nutricional en los adultos mayores se halló con el Índice de Masa Corporal (IMC) y se clasificaron en diferentes estadios según las tablas de clasificación de la sociedad española de nutrición parenteral y enteral (SENPE -2010) (14).

Para los niños se calcularon los indicadores: peso para la edad $(P / E)$, talla para la edad $(T / E)$, peso para la talla $(P / T)$ e IMC. Se tomaron como referencia las tablas publicadas en conjunto por el Instituto de Bienestar familiar (ICBF) y el Instituto Nacional de Salud (INS) (15). En las gestantes se empleó IMC ubicándolo en la gráfica ATALAH; según semana de gestación (16).

Estudio de marcadores hematológicos y bioquímicos: en ayuno se tomaron $7 \mathrm{ml}$ de sangre periférica, mediante punción venosa; $3 \mathrm{ml}$ para la determinación de hematocrito (Hto) y hemoglobina ( $\mathrm{Hb}$ ), se depositó en un tubo de $13 \times 75$ $\mathrm{mm}$ que contenía ácido etildiaminotetraacético (EDTA) como anticoagulante y se procesó en capilares sin heparina que fueron centrifugados en microcentrífuga por cinco minutos a $2.500 \mathrm{rpm}$ y luego se midió el valor en la tabla de lectura estandarizada. La determinación de Hemoglobina se realizó con el método colorimétrico estandarizado de la cianometahemoglobina utilizando el reactivo de Drabkin.

Para la determinación sérica de los marcadores bioquímicos como hierro, ferritina, transferrina y proteína $C$ reactiva $(P C R)$ se utilizaron los otros $4 \mathrm{ml}$ se sangre que fueron depositados en tubo sin anticoagulante de $13 \times 100 \mathrm{~mm}$ y posteriormente centrifugados. El hierro y la transferrina se procesaron por método colorimétrico, la ferritina y la PCR por método turbidimétrico, empleando reactivos ${ }^{\circledR}$ Biosystem. El 
equipo automatizado empleado para las mediciones fue el A15 de Biosystem.

Estudio parasitológico: se solicitó una muestra de heces fecales a cada uno de los participantes en el estudio la cual fue conservada en formol-éter al 10\% y posteriormente llevada al Laboratorio de Microbiología de la Facultad de Ciencias de la Salud de la Universidad Pedagógica y Tecnológica de Colombia (UPTC) para su procesamiento. A cada muestra se le hizo un análisis por examen directo con lugol al $1 \%$, solución salina al 0,83 \% y por técnica de concentración con formol-eter (técnica de Ritchie) (17).

Intervención: después de realizadas todas las mediciones los participantes fueron evaluados por el médico y los que tuvieron parásitos intestinales, recibieron tratamiento antiparasitario y posterior a este, recibieron un suplemento nutricional.

Análisis estadístico: los datos obtenidos de las encuestas, los parámetros antropométricos, marcadores bioquímicos, hematológicos y parásitos intestinales fueron organizados en tablas electrónicas en el programa Excel (Microsoft XP 2007), luego se relacionaron las variables frente a los resultados obtenidos y se realizó un análisis estadístico. Para el análisis estadístico se utilizaron los programas SPSS, versión 11.5, y Epinfo. 3.5.4 Se hizo un análisis de varianza, matriz de correlación y pruebas T de diferencias de medias, para determinar si hubo diferencia significativa para cada uno de los grupos y se utilizó la prueba de scheffe para determinar cuál de los grupos fue estadísticamente igual o diferentes. Para todos los cálculos se trabajó con una significancia del $5 \%$, lo que equivale a una confianza del $95 \%$.

\section{RESULTADOS}

Encuesta: los promedio de edad fueron 73, 2 y 25 años para los adultos mayores, los niños y las gestantes respectivamente, provenientes principalmente del área rural con niveles 1 y 2 según puntaje SISBEN, afiliados al régimen subsidiado, escasa escolaridad, con ocupaciones como hogar y agricultura para las gestantes y los adultos mayores.

Se determinó que los adultos mayores consumían poco suplemento nutricional, consumían tres comidas principales, 45.1\% refirió buen estado de salud y el resto enfermedades como: hipertensión, y diabetes. Las medidas higiénico sanitarias en sus casas eran todavía deficientes por falta de cubrimiento de servicios públicos básicos.

Las madres manifestaron que los niños recibían una buena alimentación, según, suministro de suplementos y lactancia materna exclusiva hasta los seis meses. Con viviendas en ladrillo que no cuentan con todos los servicios públicos básicos, $43,5 \%$ cuenta con luz, agua, acueducto y alcantarillado. La disposición de aguas residuales, 54,3\% lo hace por medio del alcantarillado, el porcentaje restante lo hace en fosas o letrinas, 30,4\% lo hace al aire libre; la disposición de basuras $32 \%$ la hace al aire libre o es quemada, el agua proviene principalmente del acueducto.

Las gestantes en su mayoría acuden a los controles prenatales, tienen en promedio 1.2 hijos; $28,57 \%$ tiene un nivel educativo técnico, ocupación ama de casa, su alimentación se basa en las tres comidas principales, además todas consumían ácido fólico y $29 \%$ había sufrido infecciones urinarias. El $85 \%$ de las viviendas estaban construidas en ladrillo, con piso de cerámica, hay un buen cubrimiento de los servicios públicos básicos.

Medidas antropométricas: el 46,2\% de los adultos mayores se encuentran eutróficos, (IMC se encuentra entre 22-27). En los IMC bajo 22, se encontró un 2,2\% de desnutrición leve
(IMC 17-18,4) y 13,2\% de peso insuficiente (IMC 18,5-21,9). Respecto a los niveles de IMC superiores a 27 , se encontró que $22 \%$ tenían sobrepeso, $12,1 \%$ obesidad tipo I y $4,4 \%$ obesidad tipo II.

En el indicador peso para la talla se encontró que 69,6\% de niños presentaron peso adecuado para la talla, 15,2\% tuvieron sobrepeso u obesidad y 15,2\% algún riesgo de desnutrición aguda. En el indicador de talla para la edad, 48\% de los niños tuvieron talla adecuada para la edad que registran; 30\% están en riesgo de retraso en el crecimiento y $22 \%$ tuvieron retraso en el crecimiento. En el peso para la edad (P/E): 52\% de los niños eran eutróficos, $13 \%$ tenían sobrepeso u obesidad. Con respecto a la desnutrición, se encontraron 35\% de los niños en riesgo de desnutrición global. El indicador Índice de Masa Corporal (IMC) mostró que $76 \%$ de los niños eran eutróficos, $15 \%$ con sobrepeso y $9 \%$ obesidad.

En cuanto al grupo de gestantes se encontró que; $57 \%$ tenían peso normal para las semanas de gestación y las restantes se clasificaron como obesidad, sobrepeso y bajo peso.

Marcadores hematológicos y bioquímicos: se encontró anemia en $26.4 \%$ de los adultos mayores; en 14,3\% mujeres y en $12,1 \%$ hombres. La mayor parte de las anemias encontradas eran anemia leve en $21 \%$, anemia moderada en $5,4 \%$ y finalmente $73,6 \%$ de los adultos mayores tenían concentraciones normales de hemoglobina.

En los niños se encontró anemia en 14,9\%, donde 6,4\% fueron niñas y $8,5 \%$ niños. La mayor parte de la anemia encontrada fue anemia leve $(8,7 \%)$ y anemia moderada $(6,2 \%)$. En $85,1 \%$ de los niños se encontraron normales las concentraciones de hemoglobina.

No se encontró gestantes con el diagnóstico de anemia.

Para la medición de los marcadores bioquímicos se desagregó la información según género y edad, dado que los valores de referencia difieren de acuerdo a estas dos variables. En el caso de los hombres con edades comprendidas entre los 50 y 64 años de edad, midiendo las concentraciones de ferritina, se evidenció un $16,7 \%$, mientras que para los mayores de 65 años este porcentaje fue 26,1\%, para los demás marcadores bioquímicos no se encontraron deficiencias representativas.

En el caso de las mujeres, para ambos grupos de edad se observaron deficiencias en los niveles de hierro y transferrina, siendo más marcadas en el primero de ellos, dado que $23,5 \%$ de las menores de 64 años estaba bajo los valores de referencia y las mayores de 65 años de edad, 11,1\%. Para el indicador de Ferritina, no se encontraron valores alterados y para la PCR como indicador de fase aguda, sólo 17,6\% de la población presentaba valores aumentados, que sugieren procesos inflamatorios o infecciosos.

En niños se encontró que $31,9 \%$ presentaron concentraciones de hierro inferiores a $50 \mathrm{ug} / \mathrm{L}$ de los cuales $14,9 \%$ corresponden a las niñas y 17,0\% a los niños. Con relación a los depósitos de hierro, establecidos por las concentraciones de ferritina se encontró que $21,3 \%$ de los niños y niñas tenían disminuidos los depósitos de hierro, con 14,9\% niñas y 6,4\% de los niños. Midiendo las concentraciones de transferrina se encontró que $8,5 \%$ niños y niñas tenían concentraciones disminuidas, de los cuales 6,4\% fueron niñas y 2,1\% niños. La medición de las concentraciones de la proteína $\mathrm{C}$ reactiva, como indicador de fase aguda, permitió evidenciar que $29,8 \%$ de los niños y niñas estaban atravesando por un proceso infeccioso o inflamatorio.

En el grupo de gestantes, no se encontró ninguna alteración en los marcadores bioquímicos analizados.

En los adultos hubo una fuerte relación directa entre las 
concentraciones de hemoglobina y hematocrito ( $r$ 0,996), la cual que fue estadísticamente significativa $(p<0,001)$. La hemoglobina se relacionó directamente de manera moderada, con la ferritina $(r 0,281)$, relación estadísticamente significativa $(p<0,01)$. El hematocrito se relacionó de forma directa, moderadamente, con la ferritina $(r 0,287)$, relación que fue estadísticamente significativa $(p<0,05)$. La ferritina y la transferrina se relacionaron inversamente de manera moderada $(r-0,294)$, relación estadísticamente significativa $(p<0,01)$. Se determinó la relación entre el índice de masa corporal y la proteína $C$ reactiva los cuales tienen una alta relación directa $(r 0,317)$ esta relación fue estadísticamente significativa $(p<0,001)$ (tabla 1).

En las gestantes hubo una fuerte relación directa entre las concentraciones de hemoglobina y hematocrito ( $r$ 0.956), relación estadísticamente significativa $(p<0.001)$. Las concentraciones de hierro se relacionaron directamente con las de ferritina $(r-0.931)$, relación estadísticamente significativa $(p<0,01)$.

En los niños y niñas también hubo una fuerte relación directa entre las concentraciones de hemoglobina y hematocrito ( $r$ 0.946), relación que fue estadísticamente significativa $(p<0.001)$. Se encontró que las concentraciones de hierro se relacionaron inversamente, de forma moderada, con la proteína $C$ reactiva $(r-0.440)$, relación estadísticamente significativa $(p<0,05)$ (tabla 2).

Parasitismo intestinal: de los 144 participantes estudiados estaban parasitados 65,3\%; todos los grupos fueron susceptibles a la infección, los adultos mayores fueron los más afectados $(75,8 \%)$, seguidos por los niños menores de cinco años (50\%) y las mujeres gestantes (28,6\%). El 34,7\% fueron negativos para parásitos intestinales. Dentro de las especies parasitarias mayor identificadas estuvieron en su orden: Entamoeba coli, Blastocystis hominis, Endolimax Nana y Entamoeba histolytica /E. dispar. Sin embargo cada resultado no es excluyente, es decir, una misma persona puede presentar uno o más parásitos, encontrándose algunos casos de poliparasitismo en los 3 grupos. La prevalencia de parasitismo intestinal global en los tres grupos de edad analizados fue 65,3\% (tabla 3).

Se realizaron pruebas de asociación, para determinar posibles factores de riesgo (antecedentes nutricionales, hábitos de higiene) sin embargo no se encontró evidencia significativa en las variables que fueron objeto de estudio.

\section{DISCUSIÓN}

La encuesta realizada mostró que la población en su gran mayoría pertenecía a los niveles 1 y 2, del SISBEN afiliados al régimen subsidiado. El $67 \%$ de la población vive en áreas rurales, desempeñando labores agrícolas y del hogar. En cuanto a las características de la vivienda, son construidas por materiales muy precarios como el adobe y pisos de tierra, lo cual hace que tengan un mayor contacto con superficies en donde habitan los parásitos que posteriormente infectan a los moradores; otro factor es que menos de la mitad de estos hogares

\section{TABLA 1}

Correlaciones de Pearson para adultos mayores.

\begin{tabular}{|c|c|c|c|c|c|c|c|c|}
\hline & & $I M C$ & $\mathrm{HB}$ & HTO & HIERRO & FERRITINA & TRANSFERRINA & PCR \\
\hline \multirow[t]{3}{*}{ IMC } & Correlación de Pearson & 1 & $-0,014$ & $-0,024$ & $-0,078$ & $-0,014$ & 0,066 &, $317^{* *}$ \\
\hline & Sig. (bilateral) & & 0,897 & 0,828 & 0,471 & 0,901 & 0,542 & 0,003 \\
\hline & $\mathrm{N}$ & 87 & 87 & 87 & 87 & 87 & 87 & 87 \\
\hline \multirow[t]{3}{*}{ HB } & Correlación de Pearson & $-0,014$ & 1 &, $996^{* *}$ & 0,142 &, $281^{* *}$ & $-0,078$ & $-0,02$ \\
\hline & Sig. (bilateral) & 0,897 & & 0 & 0,189 & 0,008 & 0,472 & 0,858 \\
\hline & $\mathrm{N}$ & 87 & 87 & 87 & 87 & 87 & 87 & 87 \\
\hline \multirow[t]{3}{*}{ HTO } & Correlación de Pearson & $-0,024$ &, $996^{* *}$ & 1 & 0,146 &, $287^{* *}$ & $-0,085$ & $-0,016$ \\
\hline & Sig. (bilateral) & 0,828 & 0 & & 0,177 & 0,007 & 0,432 & 0,881 \\
\hline & $\mathrm{N}$ & 87 & 87 & 87 & 87 & 87 & 87 & 87 \\
\hline \multirow[t]{3}{*}{ HIERRO } & Correlación de Pearson & $-0,078$ & 0,142 & 0,146 & 1 & 0,134 & 0,005 & $-0,162$ \\
\hline & Sig. (bilateral) & 0,471 & 0,189 & 0,177 & & 0,218 & 0,966 & 0,134 \\
\hline & $\mathrm{N}$ & 87 & 87 & 87 & 87 & 87 & 87 & 87 \\
\hline \multirow[t]{3}{*}{ FERRITINA } & Correlación de Pearson & $-0,014$ &, $281^{* *}$ &, $287^{* *}$ & 0,134 & 1 &,$- 294^{* *}$ & $-0,09$ \\
\hline & Sig. (bilateral) & 0,901 & 0,008 & 0,007 & 0,218 & & 0,006 & 0,405 \\
\hline & $\mathrm{N}$ & 87 & 87 & 87 & 87 & 87 & 87 & 87 \\
\hline \multicolumn{9}{|l|}{ TRANSFE } \\
\hline \multirow[t]{3}{*}{ RRINA } & Correlación de Pearson & 0,066 & $-0,078$ & $-0,085$ & 0,005 &,$- 294^{* *}$ & 1 & 0,076 \\
\hline & Sig. (bilateral) & 0,542 & 0,472 & 0,432 & 0,966 & 0,006 & & 0,483 \\
\hline & $\mathrm{N}$ & 87 & 87 & 87 & 87 & 87 & 87 & 87 \\
\hline \multirow[t]{3}{*}{ PCR } & Correlación de Pearson &, $317^{* *}$ & $-0,02$ & $-0,016$ & $-0,162$ & $-0,09$ & 0,076 & 1 \\
\hline & Sig. (bilateral) & 0,003 & 0,858 & 0,881 & 0,134 & 0,405 & 0,483 & \\
\hline & $\mathrm{N}$ & 87 & 87 & 87 & 87 & 87 & 87 & 87 \\
\hline
\end{tabular}

**. La correlación es significativa al nivel 0,01 (bilateral). 
cuentan con un alcantarillado y recolección de basuras, debido a esto, la población utiliza fosas sépticas para el manejo de las aguas residuales y al vertimiento de los desechos sólidos al aire libre, lo cual son también factores que se suman a la alta prevalencia de parásitos $(18,19)$.

En este estudio se encontraron varios factores de riesgo para sufrir alteraciones nutricionales como bajo nivel de ingresos, escasa escolaridad, dificultad en el acceso a los servicios de salud, creencias y costumbres alimentarias inadecuadas, ignorancia sobre una buena nutrición y preparación inadecuada de los alimentos.

Los resultados del estudio confirman los presentados

TABLA 2

Correlaciones de Pearson para niños y niñas menores de cinco años.

\begin{tabular}{|c|c|c|c|c|c|c|c|c|}
\hline & & IMC & $\mathrm{HB}$ & HTO & HIERRO & FERRITINA & TRANSFERRINA & A PCR \\
\hline IMC & $\begin{array}{l}\text { Correlación de Pearson } \\
\text { Sig. (bilateral) } \\
\text { N }\end{array}$ & 45 & $\begin{array}{c}0,097 \\
0,525 \\
45\end{array}$ & $\begin{array}{c}-0,029 \\
0,849 \\
45\end{array}$ & $\begin{array}{c}0,03 \\
0,844 \\
45\end{array}$ & $\begin{array}{l}0,043 \\
0,779 \\
45\end{array}$ & $\begin{array}{c}0,215 \\
0,155 \\
45\end{array}$ & $\begin{array}{c}-0,127 \\
0,405 \\
45\end{array}$ \\
\hline HB & $\begin{array}{l}\text { Correlación de Pearson } \\
\text { Sig. (bilateral) } \\
\text { N }\end{array}$ & $\begin{array}{c}0,097 \\
0,525 \\
45\end{array}$ & 45 & $\begin{array}{l}946^{* *} \\
0 \\
45\end{array}$ & $\begin{array}{c}0,26 \\
0,085 \\
45\end{array}$ & $\begin{array}{l}0,195 \\
0,2 \\
45\end{array}$ & $\begin{array}{c}-0,063 \\
0,681 \\
45\end{array}$ & $\begin{array}{c}-0,089 \\
0,563 \\
45\end{array}$ \\
\hline HTO & $\begin{array}{l}\text { Correlación de Pearson } \\
\text { Sig. (bilateral) } \\
\text { N }\end{array}$ & $\begin{array}{c}-0,029 \\
0,849 \\
45\end{array}$ & $\begin{array}{c}946^{* *} \\
0 \\
45\end{array}$ & $\begin{array}{c}1 \\
45\end{array}$ & $\begin{array}{l}330^{*} \\
0,027 \\
45\end{array}$ & $\begin{array}{l}0,131 \\
0,391 \\
45\end{array}$ & $\begin{array}{l}-0,058 \\
0,707 \\
45\end{array}$ & $\begin{array}{c}-0,082 \\
0,591 \\
45\end{array}$ \\
\hline HIERRO & $\begin{array}{l}\text { Correlación de Pearson } \\
\text { Sig. (bilateral) } \\
\text { N }\end{array}$ & $\begin{array}{c}0,03 \\
0,844 \\
45\end{array}$ & $\begin{array}{c}0,26 \\
0,085 \\
45\end{array}$ & $\begin{array}{c}, 330^{*} \\
0,027 \\
45\end{array}$ & $\begin{array}{c}1 \\
45\end{array}$ & $\begin{array}{c}-0,141 \\
0,356 \\
45\end{array}$ & $\begin{array}{c}-0,052 \\
0,734 \\
45\end{array}$ & $\begin{array}{c}-, 440^{* *} \\
0,003 \\
45\end{array}$ \\
\hline FERRITINA & $\begin{array}{l}\text { Correlación de Pearson } \\
\text { Sig. (bilateral) } \\
\text { N }\end{array}$ & $\begin{array}{c}0,043 \\
0,779 \\
45\end{array}$ & $\begin{array}{c}0,195 \\
0,2 \\
45\end{array}$ & $\begin{array}{c}0,131 \\
0,391 \\
45\end{array}$ & $\begin{array}{l}-0,141 \\
0,356 \\
45\end{array}$ & $\begin{array}{c}1 \\
45\end{array}$ & $\begin{array}{c}-, 341^{*} \\
0,022 \\
45\end{array}$ & $\begin{array}{l}0,191 \\
0,209 \\
45\end{array}$ \\
\hline $\begin{array}{l}\text { TRANSFE } \\
\text { RRINA }\end{array}$ & $\begin{array}{l}\text { Correlación de Pearson } \\
\text { Sig. (bilateral) } \\
\text { N }\end{array}$ & $\begin{array}{l}0,215 \\
0,155 \\
45\end{array}$ & $\begin{array}{c}-0,063 \\
0,681 \\
45\end{array}$ & $\begin{array}{c}-0,058 \\
0,707 \\
45\end{array}$ & $\begin{array}{c}-0,052 \\
0,734 \\
45\end{array}$ & $\begin{array}{l}-, 341^{*} \\
0,022 \\
45\end{array}$ & $\begin{array}{c}1 \\
45\end{array}$ & $\begin{array}{c}-0,225 \\
0,138 \\
45\end{array}$ \\
\hline PCR & $\begin{array}{l}\text { Correlación de Pearson } \\
\text { Sig. (bilateral) } \\
\text { N }\end{array}$ & $\begin{array}{c}-0,127 \\
0,405 \\
45\end{array}$ & $\begin{array}{c}-0,089 \\
0,563 \\
45\end{array}$ & $\begin{array}{c}-0,082 \\
0,591 \\
45\end{array}$ & $\begin{array}{c}-, 440^{* *} \\
0,003 \\
45\end{array}$ & $\begin{array}{c}0,191 \\
0,209 \\
45\end{array}$ & $\begin{array}{c}-0,225 \\
0,138 \\
45\end{array}$ & 45 \\
\hline
\end{tabular}

TABLA 3

Prevalencia de parásitos intestinales.

\begin{tabular}{|c|c|c|c|c|c|c|c|}
\hline \multirow[t]{2}{*}{ VARIABLES } & \multirow[t]{2}{*}{ TIPO DE PARASITO } & \multicolumn{2}{|c|}{ ADULTOS MAYORES } & \multicolumn{2}{|c|}{ NIÑOS } & \multicolumn{2}{|c|}{ MUJERES GESTANTES } \\
\hline & & $\mathrm{n}$ & $\%$ & $\mathrm{n}$ & $\%$ & $n$ & $\%$ \\
\hline \multirow{4}{*}{$\begin{array}{l}\text { PARASITOS } \\
\text { PATOGENO }\end{array}$} & Quistes de Entamoeba & 8 & 8,79 & 4 & 8,70 & 1 & 14,29 \\
\hline & Shistolytica /E. dispar & & & & & & \\
\hline & Quistes de Giardia intestinalis & 0 & 0,00 & 3 & 6,52 & 0 & 0,00 \\
\hline & $\begin{array}{l}\text { Huevos de Ancylostoma } \\
\text { duodenale }\end{array}$ & 0 & 0,00 & 1 & 2,17 & 0 & 0,00 \\
\hline PARASITOS & Quistes de Entamoeba coli & 38 & 41,76 & 15 & 32,61 & 2 & 28,57 \\
\hline \multirow[t]{4}{*}{ COMENSALES } & Quistes de Blastocystis hominis & 30 & 32,97 & 8 & 17,39 & 0 & 0,00 \\
\hline & Quistes de Endolimax nana & 17 & 18,68 & 5 & 10,87 & 2 & 28,57 \\
\hline & Quistes de lodamoeba butschilii & 8 & 8,79 & 1 & 2,17 & 0 & 0,00 \\
\hline & Quistes de Chilomastix mesnilii & 1 & 1,10 & 0 & 0,00 & 0 & 0,00 \\
\hline \multicolumn{2}{|c|}{ Negativo para parásitos intestinales } & 22 & 24,18 & 23 & 50 & 5 & 71,43 \\
\hline
\end{tabular}


en la última encuesta Nacional de Calidad de vida de 2013 realizada por el DANE, para la población Colombiana rural: donde 92,6\% cuentan con el servicio de luz eléctrica, 90,2\% de la población no cuentan con cobertura de gas domiciliario, sólo 58,7 \% cuentan con el servicio de acueducto-agua potable comparado con $96,8 \%$ de cubrimiento en las áreas urbanas, $76,0 \%$ tampoco cuenta con servicio de recolección de basuras y solo 5,5\% no cuentan con ningún tipo de servicio público (20).

En Colombia se estima que los hogares con nivel 1 y 2 del SISBEN presentan prevalencias de inseguridad alimentaria sobre $60 \%$ comparada con niveles superiores $(21,22)$, tal como se reportó en este estudio, cuyos participantes pertenecían a los nivel 1 y 2 del SISBEN, vivían en condiciones económicas precarias y no consumían con regularidad frutas ni verduras.

En la encuesta de hábitos de alimentación las gestantes expresaron la importancia de consumir frutas y verduras dentro de su alimentación diaria para el adecuado desarrollo del bebé, aunque no siempre las incluyen y a veces prefieren consumir alimentos de paquete y bebidas azucaradas como gaseosas o refrescos, sin estimar que le causan daño al feto en gestación $(23,24)$. Al igual que en otros estudios, se pudo determinar que las gestantes tienden al sobrepeso y a la obesidad por sobrealimentación y malas creencias alimentarias en la etapa de la gestación, donde la tendencia es a alimentarse por dos y asociar bebes obesos con buena nutrición $(25,26)$.

En los niños se pudo evidenciar que la mayoría (90\%) recibió lactancia materna exclusiva hasta los seis meses, lo que constituye un factor protector para evitar o disminuir el riesgo de desnutrición. Según la ENSIN 2010, en Colombia la duración total de la lactancia materna es 14,9 meses y es común que este tiempo se prolongue en las mujeres residentes de las áreas rurales, con menor nivel de educación y menor nivel de SISBEN (6). Según la información aportada por la madre, $26 \%$ fueron niños con bajo peso al nacer que se relaciona directamente con madres menores de 20 años, de un nivel socioeconómico bajo, que no asisten a controles prenatales o que no reciben suplementación con micronutrientes, como lo soportan otros estudios. $(27,28)$. En su mayoría, además de las tres comidas principales, los niños reciben comida de media mañana y comida de media tarde, sus madres refieren conocer la importancia de incluir en su dieta, lácteos, frutas y verduras.

En la clasificación nutricional por el IMC, se encontró que $15,4 \%$ de los ancianos estaban en un estado de desnutrición como resultado de una ingesta de alimentos insuficiente para satisfacer las necesidades de energía alimentaria, de una absorción deficiente y/o de un uso biológico deficiente de los nutrientes consumidos $(29,30)$. La desnutrición en ancianos se relaciona con múltiples consecuencias, como: alteración en la función muscular, disminución de la masa ósea, disfunción inmunitaria, anemia, repercusión en el estado cognitivo, pobre cicatrización, pobre recuperación posterior a una cirugía, incremento del riesgo de institucionalización, mayor estancia hospitalaria, fragilidad, mortalidad y reducción en la calidad de vida $(31,32)$.

También se encontró que 38,5\% de la población analizada, tenía IMC por encima del valor normal. El sobrepeso y la obesidad son el sexto factor de riesgo de defunción en el mundo. Cada año fallecen alrededor 3,4 millones de personas adultas como consecuencia del sobrepeso o la obesidad. Además, $44 \%$ de la carga de diabetes, $23 \%$ de las cardiopatías isquémicas y entre $7 \%$ y $41 \%$ de la carga de algunos cánceres son atribuibles al sobrepeso y la obesidad $(33,34)$. Hace pocos años se introdujo el término "doble carga nutricional" para referirse al fenómeno de superposición alimentaria en la comunidad, especialmente en los países subdesarrollados $(35,36)$.

Se encontró que $8,7 \%$ de los niños estaban en riesgo de sufrir desnutrición aguda, mientras 6,5\% presentaba desnutrición aguda y aguda severa. La prevalencia de desnutrición crónica fue $22 \%$ comparada con la prevalencia nacional del $13,2 \%$ reportada en la ENSIN-2010 (6). Estos resultados sugieren que a pesar de los esfuerzos que se realizan por las entidades gubernamentales en mantener las cifras reducidas, en el departamento de Boyacá las cifras aún persisten; lo cual se correlaciona con lo reportado por el sistema de vigilancia alimentaria y nutricional de Boyacá (SISVAN) con cifras de $12,3 \%, 5,5 \%$ y $18,1 \%$ respectivamente (37-39).

La anemia en los adultos mayores generalmente está asociada a las pérdidas de sangre, el estudio The Third National Health and Nutrition Examination Survey (NHANES III, 1988 to 1994) (40) estableció una prevalencia de anemia en los adultos mayores americanos del $10.6 \%$, en nuestro estudio la prevalencia de anemia de la misma población fue $26.37 \%$ más del doble de la encontrada en Estados Unidos. Es probable, según Muhammad y Pitchumoni, tenga como causa lesiones del tracto gastrointestinal (41).

La hepcidina, péptido con funciones de hormona, regula las concentraciones de hierro en la circulación inhibiendo la proporción de hierro que se exporta de los enterocitos, macrófagos y hepatocitos. En la fase aguda se sabe que se incrementa la producción de algunas citoquinas que incluyen la interluquina $6,1 \beta$, factor de necrosis $\alpha$, interferón gama; la circulación y expresión de hepcidina está relacionada con las proteínas c reactiva, alfa 1 acido glicoproteína $(42,43)$. Estudios han demostrado la asociación entre obesidad, inflamación y disminución de los depósitos de hierro (44-46). Este estudio evidenció la relación directa entre el IMC y la proteína $C$ reactiva, obteniendo un $r=0.317$, estadísticamente significativa $(<0.005)$. Según los resultados obtenidos podemos decir que la alta prevalencia de sobrepeso y obesidad en la población adulta trae como consecuencia la disminución en las concentraciones de hierro y por tanto la anemia; como lo reportado en recientes estudios de Tussing-Humphreys et al $(47,48)$.

La disminución de los depósitos de hierro en los niños menores de 5 años fue $21.27 \%$, prevalencia menor que la encontrada por el Instituto Nacional de Salud y el Ministerio de Salud en el estudio realizado entre 1995 y 1996 (58\%) y que la encontrada por Castro y Vega en el análisis de disparidades por anemia nutricional en Colombia, 2005 (49,50). La carencia de hierro es la causa más común de anemia, pero pueden causarla otras carencias nutricionales (folato, vitamina B12 y vitamina A), la inflamación aguda, crónica, las parasitosis y las enfermedades hereditarias o adquiridas que afectan a la síntesis de hemoglobina y a la producción o la supervivencia de los eritrocitos. La suplementación con hierro es la estrategia más utilizada para disminuir la prevalencia de anemia $(51,52)$.

Se observó una fuerte relación directa entre las concentraciones de hemoglobina y hematocrito, hematocrito y concentraciones de hierro y adicionalmente, el hierro se relacionó inversamente, de forma moderada, con la proteína $C$ reactiva. Estos resultados corroboran la forma en que se distribuye el hierro en las proteínas de transporte y de depósito, como son la transferrina y ferritina respectivamente; como la influencia que tiene la fase aguda en la disminución de las concentraciones de hierro, como la demostró Tussing-Humphreys et al $(47,48)$.

Las infecciones parasitarias intestinales por helmintos y protozoos son las más comunes en América Latina y han sido estimadas en su impacto sobre la salud pública; sin embargo, en la 
última década, se ha incrementado aún más su reconocimiento como un importante problema en las diferentes comunidades (53-56). Su predominio es cada vez más alto en los estratos bajos de la sociedad, en donde existen deficiencias sanitarias y marginalidad; estas infecciones se producen en el humano cuando sus hábitos y costumbres se encuentran con los ciclos de vida de los helmintos y protozoos $(57,58)$. El presente estudio encontró en los adultos mayores el parásito patógeno Entamoeba histolytica/E. dispar, pero no todas las personas que tienen el parásito desarrollan enfermedad ya que unas poseen Entamoeba histolytica y otras E. dispar que no es patógena y la observación microscópica no es una herramienta adecuada para el diagnóstico de la amebiasis, es útil como un método para la identificación de amebas del complejo E. histolytica/E. dispar, sobre todo en los grandes estudios epidemiológicos (17). También se encontraron parásitos comensales como Entamoeba coli que se transmite en forma de quiste viable que llega a la boca por contaminación fecal y se deglute, se localiza en la luz intestinal (intestino grueso) (17). Blastocystis hominis del cual su patogenicidad ha sido discutida por largos años ya que algunos investigadores consideran que carece de importancia clínica pero tiene importancia epidemiológica; la patogenicidad puede depender del número de parásitos presentes en las muestras de heces, de la presencia de otros microorganismos concomitantes y del estado inmunológico del paciente (59). El grupo de los niños menores de cinco años tuvo una presencia similar de parásitos a los adultos mayores exceptuando un caso de Ancylostoma duodenale que se asocia a la vida rural y a la manipulación de tierra (60-62). Sólo una mujer gestante presentó parásitos intestinales patógenos Entamoeba histolytica/E. dispar lo cual indica que han tenido en cuenta las medidas higiénico sanitarias y una buena adherencia a los controles prenatales y sus recomendaciones.

La blastocistosis es una parasitosis intestinal que tiene una alta prevalencia tanto en el ámbito nacional como internacional, por lo cual, Blastocystis hominis fue uno de los parásitos más encontrados en adultos mayores como en niños menores de cinco años, similar a lo reportado en otras investigaciones $(57,59,63)$, dicho parásito sigue siendo de patogenicidad discutida pero su prevalencia es aun alta. El consumo de agua potable no hervida, con ausencia de medidas adecuadas de saneamiento básico, son factores de riesgo determinantes importantes en la prevalencia de $\mathrm{B}$. hominis, especialmente en niños. La Entamoeba histolytica/E. dispar, como lo han reportado otras investigaciones $(57,59,64)$; en el momento que invade la mucosa intestinal causa úlceras a nivel del ciego y en la región sigmoidea, puede penetrar las capas muscular del intestino o atravesar la serosa perforando el peritoneo y afectar otros órganos como hígado, pulmones, piel y cerebro (17).

En la comunidad estudiada el hallazgo de comensales como Entamoeba coli, Endolimax nana y lodamoeba butschilii con una frecuencia similar a la encontrada en otros estudios realizados mundialmente $(64,65)$, hecho importante debido a que es un indicador de fecalismo, malos hábitos higiénicos, lo que permite inferir su elevada probabilidad de transmisión y aunque estas especies no son patógenas y no tienen gran impacto clínico, son de suma importancia reportarlas por su relevancia epidemiológica, ya que muestra que el individuo parasitado puede ser un foco latente de transmisión y para evitar infectarse se debe tener una óptima higiene personal (66).

En el presente estudio hubo un predominio de protozoos sobre helmintos lo cual coincide con otros estudios, debido a las características que tienen los quistes de los protozoos; que son estables en el medio ambiente, efectivos aún en bajas infecciones y que no son destruidos por el cloro en concentraciones utilizadas para la potabilización del agua $(9,10,57,65,66)$.

\section{CONCLUSIONES}

- La población estudiada presenta varios factores de riesgo de alteraciones nutricionales y parasitismo intestinal como son el acceso restringido a alimentos por bajos ingresos, bajo nivel de escolaridad, falta en el suministro de agua potable, servicios higiénicos inadecuados, alcantarillado insuficiente y falta de eliminación de desechos sólidos y líquidos entre otros.

- Las medidas higiénico sanitarias aun son deficientes por falta de cubrimiento de los servicios básicos y a pesar de haber encontrado un alto porcentaje de parásitos intestinales no se evidenció asociación estadísticamente significativa con la desnutrición y los factores predisponentes encontrados en el estudio.

- La prevalencia de parásitos intestinales en los grupos estudiados fue $67,8 \%$, predominando los protozoos y los parásitos no patógenos. Se encontró mayor número de parásitos intestinales en niños y adultos mayores que en mujeres gestantes.

\section{RESUMEN}

El objetivo del estudio fue establecer el estado nutricional, el parasitismo intestinal y sus factores de riesgo en mujeres gestantes, adultos mayores y niños menores de 5 años. A cada uno de los participantes se le aplicó una encuesta, valoración del estado nutricional (peso y talla), medición de indicadores como hemoglobina, hierro sérico, ferritina, transferrina, proteína $C$ reactiva, y búsqueda de parásitos intestinales, seguido de un plan de manejo médico. Se encontraron como factores de riesgo para parasitismo intestinal, malos hábitos de higiene y falta de cubrimiento de los servicios públicos básicos. En los adultos mayores se encontró que 22\% tenían sobrepeso, 16,5\% obesidad, los niños y niñas 15,2\% tuvieron sobrepeso u obesidad y 15,2\% presentaron algún riesgo de desnutrición aguda, por otra parte presentaban prevalencias de anemia leve y moderada, 26,4\% de adultos mayores y $14,9 \%$ de los niños. La prevalencia de parásitos intestinales fue 65.3\%; encontrándose parásitos como Entamoeba coli, Blastocystis hominis y Entamoeba histolytica/E. dispar. Es importante fortalecer los programas de seguridad alimentaria para disminuir los índices de malnutrición. A pesar de haber encontrado un alto porcentaje de parásitos intestinales no se encontró asociación estadísticamente significativa con la desnutrición y los factores predisponentes.

Palabras clave: población vulnerable, parasitismo intestinal, malnutrición, deficiencia de hierro, anemia.

Agradecimientos y financiación: Proyecto Universidad Pedagógica y Tecnológica de Colombia - Tunja - Boyacá.

Conflicto de intereses: ninguno declarado.

\section{BIBLIOGRAFÍA}

1. Panorama de la Seguridad Alimentaria y Nutricional en América Latina y el Caribe 2013. Hambre en América Latina y el Caribe: acercándose a los Objetivos del Milenio. Consultado en Octubre de 2014. Disponible en:www.fao.org

2. Kerry J, Parul C, Lee S, Margia A, Hongjie C, Ashika N, Christine $P$, Subarna K, Steven L, Keith P. Micronutrient Deficiencies are common in 6- to 8-Year-old children of 
rural Nepal, with prevalence estimates modestly affected by Inflammation. J Nutr. 2014; 144: 979-87.

3. Holguín E, Orozco JG. Administration of folic acid and other micronutrients to pregnant women in Colombia. Rev Panam Salud Pública. 2013; 34(2): 99-106.

4. World Health Organization. Salud de la madre, el recién nacido, del niño y del adolescente (Internet). Ginebra: WHO. Consultado en Octubre de 2014. Disponible en: http://www.who.int/maternal_child_adolescent/es/index. html.

5. Sobrino M, Gutiérrez C, Cunha AJ, Dávila M, Alarcón J. Desnutrición infantil en menores de cinco años en Perú: tendencias y factores determinantes. Rev Panam Salud Pública. 2014; 35(2): 104-12.

6. Encuesta Nacional de la Situación Nutricional en Colombia 2010. ENSIN. Encuesta dirigida por el ICBF en convenio con el Ministerio de la Protección social, Instituto Nacional de Salud y Profamilia. Colombia 2008-2010.

7. Guía de Atención de Desnutrición. República de Colombia. Ministerio de Salud. Dirección general de Promoción y Prevención. 1998.

8. Alvarado $B E$, Vásquez $L R$. Social determinants, feeding practices and nutritional consequences of intestinal parasitism in young children. Biomédica. 2006; 26(1): 82-94.

9. Bonilla DC. Asociación entre estado nutricional y la presencia de parasitosis intestinales en niños preescolares del colegio San Francisco de Asís, barrio el codito y bella vista. [Tesis]. Bogotá D.C: Pontificia Universidad Javeriana. Facultada de Ciencias. Carrera de Nutrición y Dietética; 2009.

10. Borrego BA. Influencia de factores ambientales y desnutrición en parasitosis intestinales en preescolares de centros municipales de bienestar infantil en ciudad Juárez en 2009. [Tesis]. Ciudad de Juárez: Universidad Autónoma de Ciudad Juárez. Departamento de Ciencias de la Salud; 2010.

11. Botero JH, García GM, Grisales D, Aguirre DC, Álvarez MC. Giardia intestinalis and nutritional status in children participating in the complementary nutrition program, Antioquia, Colombia, May to October 2006. Rev Inst Med Trop S. 2009; 51(3): 155-62.

12. Londoño A, Mejía S, Gómez J. Prevalencia y factores de riesgo asociados a parasitismo intestinal en preescolares de zona urbana en Calarcá, Colombia. Rev Salud Pública. 2009; 11(1): 72-81.

13. República de Colombia. Ministerio de Salud. Resolución $N^{\circ} 008430$ de 1993. Consultado en Octubre de 2014. Disponible en: http://www.unisabana.edu.co/ fileadmin/Documentos/Investigacion/comite_de_etica/ Res_8430_1993_-_Salud.pdf

14. Gregorio $P G$, Ramos $P$, Cuesta $F$, Mañas MC, Cuenllas $A$. Nutrición en el Anciano. Guía de buena práctica clínica en geriatría. Consultado en Octubre de 2014. Disponible en http:/Users/Invitado/Downloads/guia_NESTLE.pdf.html

15. Instituto Colombiano de Bienestar Familiar (ICBF). Guía técnica y operativa Sistema de Seguimiento Nutricional. 2014. Consultado en Octubre de 2014. Disponible enhttp://www.icbf.gov.co/portal/page/portal/IntranetICBF/macro_procesos/MP_misionales/G_nutricion.html

16. Atalah E, Castillo C, Castro R. Propuesta de un nuevo estándar de evaluación nutricional en embarazadas. Rev. Med. Chile. 1997; 125:1429-1436

17. Botero D. Parasitosis Humanas. 5 Edición. Medellín, Corporación para las Investigaciones Biológicas, 2012.

18. Arias J, Guzmán G, Lora F, Torres E, Gómez J. Prevalencia de protozoos intestinales en 79 niños de 2 a 5 años de edad de un hogar infantil estatal en Circasia, Quindío. Infectio 2010; 14(1):31-8.

19. Oropesa OL, Quevedo G, Leyva L, Ferra B, Ferrer I, Rodríguez N. Intervención educativa sobre parasitismo intestinal en niños de la Escuela Primaria Salvano Velazco, Bocono. Misión Médica Cubana. Coordinación Docente Estadal Trujillo. República Bolivariana Venezuela. 2010;14(1):1-12.

20. DANE. Encuesta Nacional de Calidad de Vida. Condiciones Socioeconómicas de los hogares Colombianos. SeptiembreOctubre de 2013.

21. Documento Técnico de la situación en seguridad Alimentaria y Nutricional (SAN). Organización de las Naciones Unidas para la Alimentación y la Agricultura (FAO), Proyecto UTF/ COL/039. Bogotá DC, febrero de 2013.

22. Plan Nacional de Seguridad Alimentaria y Nutricional (PNSAN) 2012-2019. Observatorio de Seguridad Alimentaria y Nutricional de Colombia, Fase I. Equipo Ministerio de Agricultura y Desarrollo Rural, Ministerio de Salud y Protección Social y Agencia de las Naciones Unidas para la Alimentación y la Agricultura-FAO. Diciembre 17 de 2012.

23. Cruz C, Cruz L, López M, González J. Nutrición y embarazo: algunos aspectos generales para su manejo en la atención primaria de salud. Rev Habanera Ciencias Med. 2012; 11(1): 168-75.

24. Horton $K$, Adetona O, Aguilar M, Cassidy B, Pfeiffer $C$, Schleicher R, Caldwell K, Needham L, Rathbun S, Vena J, Naeher L. Changes in the concentrations of biochemical indicators of diet and nutritional status of pregnant women across pregnancy trimesters in Trujillo, Peru,2004-2005. Nutri J. 2013; 12:80-6.

25. Sarmiento $O$, Ramírez $A$, Samper $B$, Pinzón P, García $S$, Olarte A, Mosquera T, Atalah E, Ojeda G, Forero Y. Nutrition in Colombian pregnant women. Public Health Nutr. 2012; 15(6): 955-63.

26. Forero N, Gamboa LF, Bedi A, Sparrow R. Child malnutrition and prenatal care: evidence from three Latin American countries. Rev Panam Salud Pública. 2014; 35(3): 163-71.

27. Ducuara P. Determinantes socio-económicas de la desnutrición global infantil en la localidad de ciudad bolívar (Bogotá, Colombia) en el año 2011. [Tesis]. Bogotá: Universidad Colegio Mayor de Nuestra Señora del Rosario. Facultad de administración. Maestría de administración en salud; 2012.

28. Chavarro D, Reyes C, Samper R, Arciniegas AJ, Gutiérrez CC. Nutritional assessment and factors associated to malnutrition in older adults: A cross-sectional study in Bogotá, Colombia. J Aging Health. 2014; 26(6): 197-207.

29. Cardona D, Segura A, Espinosa A. Mortalidad de adultos mayores por deficiencias nutricionales en los Departamentos de Colombia. Rev. Salud Pública. 2012; 14(4): 584-97.

30. García T, Villalobos JA. Malnutrición en el anciano. Parte I: desnutrición, el viejo enemigo. Med Int Mex 2012; 28(1): 57-64.

31. González J. Evaluación del estado nutricional de un grupo de adultos mayores pertenecientes al plan nueva sonrisa dispensario Santa Francisca Romana. [Tesis]. Bogotá: Pontificia Universidad Javeriana. Carrera de Nutrición y Dietética; 2011.

32. Rasheeda S, Woodsb RT. Malnutrition and quality of life in older people: A systematic review and meta-analysis. Ageing Res Rev. 2013; 12: 561- 6.

33. Villarreal E, Forero Y, Poveda E, Baracaldo C, López E. Marcadores de riesgo cardiovascular en escolares de cinco departamentos de la región oriental en Colombia. Bio- 
médica 2008; 28: 38-49.

34. Poveda E, Callas N, Baracaldo C, Castillo C, Hernández P. Concentración sérica de leptina en población escolar de cinco departamentos del centro-oriente colombiano y su relación con parámetros antropométricos y perfil lipídico. Biomédica 2007; 27: 505-14.

35. Sarmiento OL, Parra DC, González SA, González I, Forero AY, García J. The dual burden of malnutrition in Colombia. Am J Clin Nutr. 2014; 100(6): 1628S-35S.

36. Pastor GL, Manrique F, Ernert A, Bau AM. The double burden of malnutrition and its risk factors in school children in Tunja. Arch Latinoam Nutr. 2012; 62(2): 119-26.

37. Garcia S, Sarmiento OL, Forde I, Velasco T. Socio-economic inequalities in malnutrition among children and adolescents in Colombia: the role of individual, household and community-level characteristics. Public Health Nutr. 2013; 16(9): 1703-18.

38. Márquez H, García V, Caltenco M, García E, Márquez H, Villa A. Clasificación y evaluación de la desnutrición en el paciente pediátrico. Rev Medigraphic. 2012; 7(2): 59-69.

39. Quiroga EF. Death rate by malnutrition in children under the age of five, Colombia. Biomedica. 2012; 2(4): 499-509.

40. Looker AC, Dallman PR, Carroll MD, et al. Prevalence of iron deficiency in the United States. JAMA. 1997; 277: 973-6.

41. Moore JR. Ormseth M, Fuchs H. Causes and Significance of Markedly Elevated Serum Ferritin Levels in an Academic Medical Center. J Clin Rheum. 2013; 19(6): 324-8.

42. Park KH, Sawada $T$, Kosuge $T$, et al. Surgical inflammation induces hepcidin production after abdominal surgery. World J Surg 2012; 36:800-6.

43. Saraiva $B$, Soares $M$, Dos Santos $L$, Pereira $S$, Horta $P$. Iron deficiency and anemia are associated with low retinol levels in children aged 1 to 5 years. J Pediatr. 2014; 90(6): 593-9.

44. Gaffney $E$, McClung P. Inflammation and diminished iron status: mechanisms and functional outcomes. Curr Opin Clin Nutr Metab Care. 2012; 15(6): 605-13.

45. Hermoso M, Vucic V, Vollhardt C, Arsic A, Roman-Vinas B. The Effect of Iron on cognitive development and function in infants, Children and Adolescents: A systematic review. Ann Nutr Metab. 2011; 59: 154-65.

46. Manjarrés LM, Díaz A, Carriquiry A. Asociación entre la ingesta de nutrientes hematopoyéticos y el origen nutricional de la anemia en mujeres en edad fértil en Colombia. Rev Panam Salud Pública. 2012; 31(1): 68-73.

47. Tussing LM, Nemeth E, Fantuzzi G. Elevated systemic hepcidin and iron depletion in obese premenopausal females. Obesity (Silver Spring) 2010; 18: 1449-56.

48. Tussing LM, Nemeth $E$, Fantuzzi $G$, et al. Decreased serum hepcidin and improved functional iron status 6 months after restrictive bariatric surgery. Obesity (Silver Spring). 2010; 18:2010-16.

49. Castro L, Nicholls S. Deficiencia de hierro, vitamina A y prevalencia de parasitismo intestinal en la población infantil y anemia nutricional en mujeres en edad fértil, Colombia 1995-96. Instituto Nacional de Salud. 1998.

50. Vega $R$, Acosta N, Martínez J, Arrieta $R$, Estupiñán Z, Fonseca Z, Castro C Análisis de disparidades por anemia nutricional en Colombia, 2005. Rev Gerenc Polit Salud, Bogotá (Colombia). 2008; 7(15): 46-76.

51. Organización Mundial de la Salud. Concentraciones de ferritina para evaluar el estado de nutrición en hierro en las poblaciones. Sistema de Información Nutricional sobre Vitaminas y Minerales. Ginebra, Organización Mundial de la Salud, 2011. Consultado en Octubre de 2014. Disponible en:http://www.who.int/vmnis/indicators/serum_ferritin_ es.pdf.

52. Perignon $M$, Fiorentino $M$, Kuong $K$, Burja $K$, Parker $M$, Sisokhom S, Chamnan C, Berger J, Wieringa FT. Stunting, poor iron status and parasite infection are significant risk factors for lower cognitive performance in cambodian school-aged children. PLoS One. 2014; 9(11): 1-11.

53. Arencibia $H$, Lobaina J, Terán C, Legrá $R$, Arencibia A. Parasitismo intestinal en una población infantil venezolana. Medisan. 2013; 17(5): 742-8.

54. Espinosa M, Alazales M, García A. Parasitosis intestinal, su relación con factores ambientales en niños del sector "Altos de Milagro" Maracaibo. Rev Cubana Med General Integral. 2011; 27(3): 396-405.

55. Garaycochea $O$, Acosta G, Vigo N, Heringman $K$, Dyer $A$, Jerí S, Siancas $G$. Parasitismo intestinal, anemia $y$ estado nutricional en niños de la comunidad de Yantaló, San Martín, Perú. Rev Ibero-Latinoam Parasitol. 2012; 71(2): 143-51.

56. Martínez De la Ossa D, Arrieta M, Ampudia A, Fernández M, Hernández $S$, et al. Parasitosis Intestinal. Ciencia Salud Virtual. 2010; 2(1): 122-9.

57. Suescún S. Prevalencia de parásitos intestinales y factores de riesgo en escolares del colegio Chicamocha Kennedy I del municipio de Tuta, Boyacá - Colombia. Rev Univ Salud. 2013; 15(2): 218-24.

58. Pérez de la Torre L. Diagnóstico eminentemente por interrogatorio de las enteroparasitosis comunes, especialmente la giardiasis. Policlínico Comunitario Docente Este. Camagüey, Cuba. AMC. 2010; 14(2): 10-22.

59. Díaz M.A, Varengo H. T, Marini Vy Orsilles M.A. Prevalencia de Blastocystis sp en niños y adolescentes de comunas periurbanas de la ciudad de Córdoba, Argentina. Rev IberoLatinoam Parasitol 2011; 70(1): 35-41.

60. Mascarini-Serra L. Prevention of soil-transmitted helminth infection. J Glob Infect Dis. 2011; 3: 175-82.

61. Fernández J.A, Reyes $P$, López C, Moncada L., Díaz M.P. Factores asociados a la infección por geohelmintos en escolares y preescolares en La Virgen, Cundinamarca. Rev Med Sanit. 2010; 13(4): 48-52.

62. Restrepo IC, Mazo LP, Salazar ML. Montoya MN, Botero JH. Evaluación de tres técnicas coproparasitoscópicas para el diagnóstico de geohelmintos intestinales. latreia. 2013; 26(1): 15-24.

63. Manrique FG, Suescún SH. Prevalencia de parasitismo intestinal y situación nutricional en escolares y adolescentes de Tunja. Rev CES Med. 2011; 25(1):20-30.

64. Goncalves $A L$, Belizario $T L$, Pimentel JB, Penatti MP, Pedroso RS. Prevalence of intestinal parasites in preschool children in the region of Uberlandia, State of Minas Gerais, Brasil. Rev Soc Bras Med Trop. 2011; 44: 191-3.

65. Novo I, Martín A, Elcuaz $R$, Muro A, Rodríguez A, García I, Bordes A, Carranza R, Hernández M, Suárez A y Pérez J. Parasitosis en Gran Canaria (España). Estudio prospectivo multicéntrico durante un año. Rev Ibero-Latinoam Parasitol 2012; 71(1): 34-41.

66. Papier K, Williams GM, Luceres R, Ahmed F, Olveda RM, McManus DP, et al. Childhood malnutrition and parasitic helminth interactions. Clin Infect Dis. 2014; 59(2): 23443. 